\title{
The Impending 5G Era and Its Likely Impact on Society
}

\author{
Abel Nyamapfene \\ University College London, London, UK. \\ a.nyamapfene@ucl.ac.uk
}

\begin{abstract}
This paper looks at the emergence of the fifth generation of wireless networks, commonly referred to by the acronym $5 \mathrm{G}$, from a perspective informed by the literature on digital divides and digital inequality. 5G has been designed with the goal of minimising inequalities in physical access, in particular differences in access that arise as a consequence of the rural-urban divide. Together with the Internet of Things, 5G is likely to usher in a new era of economic growth, and to lead to general improvements in quality of life. However, the impact of $5 \mathrm{G}$ on socio-economic status is not so clear cut. In some instances, 5G is likely to magnify social inequality, while in other instances it is likely to narrow social inequality. $5 \mathrm{G}$ also has the potential to alter existing socio-economic hierarchies, and this paper cautions policy makers and social elites to minimise the negative impacts of attendant social transformations.
\end{abstract}

Keywords -5G, digital divide, digital inequality, Internet of Things, IoT.

\section{INTRODUCTION}

We are now currently at a stage where the predominant mode of electronic communication is through mobile technology, and whereby the predominant medium for communication is the internet [1]. This electronic communication increasingly involves both man-to-man communication as well as machine-to-machine communication. By 2020 it is expected that 50 billion devices will be connected to mobile networks, leading to a thousand-fold increase in the volume of data handled by mobile networks [2]. Fifth generation (5G) network architectures able to handle this huge amount of data from heterogeneous devices are currently being investigated $[1,2]$. At a minimum, $5 \mathrm{G}$ networks should be highly scalable with regard to the number of connected devices that they can handle, and to cater for a wide variety of services, including those services we cannot anticipate at the present moment, they should be adaptable enough to simultaneously provide a diverse range of quality of service $(\mathrm{QoS})$ requirements $[3,4]$.

It is expected that $5 \mathrm{G}$ networks should be greener than current mobile network technologies with respect to cost, energy consumption and resource utilisation, and should be available to anyone anywhere virtually all the time. To this extent, compared to current mobile networks, $5 \mathrm{G}$ networks should $[3,4]$ :

- Have the capacity to handle a 1000-fold increase in mobile data

- Support 10 to 100 times today's current data rates

- Offer at least 10 times today's current battery life for low power devices connected to the network

- Have 5 times less end-to-end latency

- Have $90 \%$ less energy usage

- Offer a perceived availability of $99.999 \%$

- Provide almost 100\% coverage irrespective of users' locations

To ensure delivery of these requirements, it is anticipated that the emerging $5 \mathrm{G}$ networks will have to comprise multiple heterogeneous technologies interworking together [2]. It is also expected that the $5 \mathrm{G}$ networks should be user-centric so as to provide a flexible, personalised service that meets end-user needs[5]. According to a 2014 survey of mobile network users by Enders Analysis, the quality of service parameters that are of most concern to users are reliability (47\%), coverage (36\%) and data speed (9\%) [6]. To meet these user concerns, 5G networks should [5]:

- Provide a continuous, uninterrupted service with minimum disruptions

- Hide any network technicalities and complexities from the user

- Offer user personalization and service differentiation

- Keep track of quality of user experience and to optimize this in an energy efficient manner.

Clearly, the network requirements for $5 \mathrm{G}$ networks have been designed with the objective of ensuring that everyone will have equal access to the network regardless of their location and their social status in the community. For instance, the requirement to provide a coverage of almost $100 \%$ in all locations indicates that people in remote rural areas will have access to the same services as people living in 
metropolitan areas. Guaranteeing a continuous uninterrupted service that has the same capacity across all locations also ensures that quality of service seizes to be location dependent.

On the basis of the proposed $5 \mathrm{G}$ network requirements, it appears that proponents of $5 \mathrm{G}$ networks believe that if $5 \mathrm{G}$ is able to deliver on these requirements, then the digital divide between social groups, and between rural and urban communities will be largely addressed. Providing equal access to a communication platform such as the $5 \mathrm{G}$ network has the potential to provide equal access to information, contacts and resources, and therefore equality of opportunity, regardless of social status. However van Dijk and Hacker [7] have recently argued that providing equal access, through for example, giving everyone a computer and a network connection, does not necessarily address the digital divide.

Rather, van Dijk and Hacker argue that the concept of a digital divide is a complex phenomenon that goes beyond equality of physical access, and that is rooted in the social inequalities inherent in modern society. This suggests that for $5 \mathrm{G}$ to deliver on its objectives, more needs to be done beyond guaranteeing an equal access network. One of the objectives of this paper is to identify those non-technical social aspects that need to be addressed to ensure that the equal access and equality of opportunity goals of $5 \mathrm{G}$ are realized.

The relationship between technology and social inequality is not static, or unidirectional. As Freeman Dyson has recently argued, the introduction of a new technology into society has the potential to address current social inequalities, and to give rise to new social inequalities that also need to be addressed [8]. This means that $5 \mathrm{G}$ has the potential to address current inequalities, and the potential to introduce new inequalities. This paper will attempt to clarify the current social inequalities that can be addressed by the introduction of 5G. In addition, an attempt will be made to identify any potential inequalities that may be spawned by the introduction of $5 \mathrm{G}$.

\section{DIGITAL DIVIDE AND DIGITAL INEQUALITY IN THE WIRELESS ERA}

Over the past twenty years the use of information and communication technology has become central to everyday life. Advances in the proliferation and use of the internet has led to an increasingly networked world, and the ability to access the internet and to use it effectively now has significant economic, social, cultural and political implications on individuals[9]. Given that access to the internet is increasingly taking place through mobile technology, this also means that how people access and use $5 \mathrm{G}$ will have consequences on their life chances. Increasingly, people are using the internet for day to day activities such as shopping, social networking, looking for jobs and business opportunities and for entertainment. With the advent of $5 \mathrm{G}$ this trend is bound to increase. This suggests that those people with unrestricted access to $5 \mathrm{G}$ and have the ability and skills to fully utilise it have a distinct advantage over those with restricted access, or those who, for a variety of reasons, are unable to fully utilise the $5 \mathrm{G}$ in pursuit of their life goals.

The term "digital divide" is often used to refer to unequal access to digital networks such as the internet, which is by far, the de facto digital communication medium for the whole world. Hargittai suggests that in practice, the term "digital divide" has been used to differentiate between those who have access to a de facto communication medium and those who do not have access [9]. She feels that this is no longer appropriate since the majority of society is now online, and all these people have varying levels of access to the internet, and use it in a variety of ways. She proposes the term "digital inequality" to capture this spectrum of differences in access and use of digital communication mediums. Given that a stated goal of $5 \mathrm{G}$ is to provide universal mobile access to the internet, it would seem that the term "digital inequality" is more descriptive of how society is likely to engage with $5 \mathrm{G}$ as opposed to the term "digital divide" with its implication of a binary divide in access.

A general perception amongst researchers is that the digital divide and digital inequality are both rooted in social inequality [7, 9-13]. When the internet first emerged in the 1990 's it was widely believed that it would help to provide a more egalitarian access to information. Contrary to this belief, however, it quickly became clear that some social groupings, notably those who were highly educated and on high incomes, were more likely to use the internet than other groupings [11]. This suggested that the internet, contrary to equalizing access to information, was actually having the opposite effect by accentuating the inequalities in society [11]. $5 \mathrm{G}$ offers the same promise of providing equal access to information channels and social networks as the Internet, and it is therefore pertinent to explore how social inequalities contribute to inequality, and how this can be mitigated.

Van Dijk and Hacker [7] identified four dimensions of digital inequality. These dimensions are a lack of appropriate experience in the new digital technology, non-possession of access technologies such as computers and network connections, lack of digital skills, and lack of significant usage opportunities. In the early 1990's when mobile technology and the internet were introduced, inexperience with the emerging technologies and non-possession of access devices were the most critical dimensions. People most affected by 
this included the elderly, those with low educational qualifications, those on low incomes, as well as those who lived in rural areas.

Once a person managed to gain easy access to the internet, then they easily acquired the necessary skills to operate in that medium. This means that those with the resources and ability to get onto the internet had a head start compared to less able people. It is therefore apparent that an individual's lack or possession of digital skills is to some extent influenced by access to the new technology, and by the person's ability to learn and master the new technology.

As a general rule the young and the educated are better able to adopt and experiment with new technologies and to use them in their day to day lives. In contrast, the elderly and less educated are less inclined to adopt new technologies. An example is the internet whereby in advanced economies the elderly are less likely to use the internet even though it has the scope to offer them a better livelihood given their restricted mobility as a result of age. If the elderly are able to access and use the Internet, then it becomes possible for them to take control of their lives by using it to undertake every day activities like banking, shopping and even getting medical advice. With advances in internet and networking technologies, including 5G, it is apparent that more and more activities are going to be accessible over the internet, thereby making access to $5 \mathrm{G}$ a necessity for modern day life. $5 \mathrm{G}$ will enable a wide variety of sensors to be connected to homes and to individuals, and this will enable doctors to monitor the health conditions of the elderly directly and in real time. Hence, it is of direct benefit to the elderly if they are sufficiently motivated to engage with emerging $5 \mathrm{G}$ technologies.

5G promises equal access to all individuals regardless of location. This means that even people in rural areas are likely to have the same level of access as people in urban areas. However, compared to urban areas, rural areas are more sparsely populated, and therefore more expensive to connect than urban areas. The task of interconnecting rural areas cannot therefore be left to private network providers as this tends to be uneconomic. Government has to contribute to this through formulating appropriate policies, giving incentives to network providers, and directly investing in network infrastructure. Just as it is the responsibility of governments to provide people with transport networks, it should also be the responsibility of government to ensure that everyone has equal access to $5 \mathrm{G}$ regardless of location.

5G also promises to make the technicalities and complexities of the underlying technology transparent to users. This is very important in reducing digital inequality since technologies that are not user-friendly often lead to access problems in practice [14]. This suggests that $5 \mathrm{G}$ users will not experience a significant jump in the look and feel of user interfaces. This can be achieved by the development of multimodal user interfaces that allow the user to interact with the network using all five senses, including voice, hand gestures and facial gazes [15]. Multimodal user interfaces reduce the need to learn complex sequences of instructions, and in this way they make complex networks more tractable. In addition, we are also witnessing the advent of intelligent personal assistants in mobile and computing devices. These personal assistants simplify man-machine interfaces by anticipating user needs and issuing commands and requests to the underlying technology to carry out various tasks on behalf of the user. Examples include Apple's Siri [16] and Google Now [17]. Intelligent personal assistants are likely to improve over time, and it is possible that by the time $5 \mathrm{G}$ is well established, they will be in routine use. This will help to realise another objective of $5 \mathrm{G}$, namely to facilitate personalised services for end users.

A key feature of $5 \mathrm{G}$ is that it enables access to the internet by both individuals and by connected devices[2, 3]. Indeed, 5G is being developed with a view to serving as the communication medium for the Internet of Things (IoT). Atzori et al. [18] define the IoT as world-wide network of interconnected objects uniquely addressable, based on standard communication protocols. They also believe that the IoT will make possible a huge range of applications, including new applications in transportation and logistics, healthcare, smart environments, and personal and social domains. Such applications have the potential to improve our quality of life by taking charge of the day to day mundane aspects of our business and social lives. A key consequence of this is that the need for extensive digital skills is likely to disappear as IoT technologies become more widely utilized.

Looking back at Van Dijk and Hacker's dimensions of digital inequality, it would appear that introduction of $5 \mathrm{G}$ is well placed to address the three dimensions directly associated with physical access and use of the network. 5G achieves this through guaranteeing these three dimensions are a lack of appropriate experience in the new digital technology, non-possession of access technologies such as computers and network connections, and a lack of digital skills. The fourth dimension, namely the lack of significant usage opportunities, requires some more in depth analysis.

\section{EQUALITY OF USAGE OPPORTUNITIES IN THE 5G ERA}

Even when equal access is guaranteed, there are still differences in people's ability to leverage a digital network to their benefit. Van Dijk [14] suggests that such an ability 
requires mastery of at least four skills, namely information, communication, strategic and content-creation skills. All these four skills are to do with the management, utilisation and creation of content on the digital network. Information skills relate to the ability to search, select and evaluate information, whilst communication skills refer to the ability to establish contacts, create viable online identities, and the ability to draw attention and give opinions [14].

Information and communication skills help to create an appropriate persona on the digital network, and the success or failure of this has a direct bearing on an individual's level of strategic skills, namely the ability to use digital networks to achieve personal and professional goals. Such skills depend to a great extent on the extent to which the individual feels at home in the digital network environment, as well as the power relationships amongst the individuals making up the network.

In normal society individuals experience varying degrees of social stratification based on gender, ethnicity, religion, and social and economic status. As society migrates to the digital domain, it is likely that such stratifications are likely to be replicated in the digital domain. After all, the digital domain becomes the main forum for social and economic interaction, and agents who are dominant in the real world will want to extend their dominance to the digital world. For instance, in a paternalistic society, privileged males may be inclined to assert their privilege over women, those who hold political and economic power may seek to maintain their hold over the instruments of socio-economic control.

However, digital networks should not be viewed as just digital replicas of physical socio-economic networks. They complement these networks, and also open up completely new channels of communication and interaction. For instance, whilst physical social networks may be constrained by physical artefacts such as country borders, digital networks easily extent beyond these physical boundaries. Such characteristics make it virtually impossible for social agents in an existing social network to maintain the same level of leverage and control over the same social agents when they move to the digital domain. For instance, whereas it is relatively easy to control access to information and communication in physical social networks, this is considerably much more difficult in a networked environment as evidenced by how the authorities in Egypt failed to put down the "Arab Spring" revolution in that country [19].

The ability to create content on digital networks gives individuals the ability to influence opinion and to profit from this. For instance, a person who sets up a blog can end up influencing a sizeable proportion of the population, and this may lead to recognition and heightened social status. However, if content creation is primarily the preserve of social elites, then digital networks may lead to social inequality being accentuated. On the other hand, if content creation is primarily the reserve of low-status people, then digital domains may lead to a narrowing of social inequality.

Blank [20] analyzed internet content creation by a random sample $(\mathrm{N}=1498)$ of British internet users in 2011. With regard to creative content, including blogs, personal websites, stories and poetry, social status had no effect at all. Existing elites and low status individuals were just as likely as not to create creative content. However, he discovered that non-elites were more likely to create social and entertainment content, whilst the elites were more likely to generate political content. Specifically, political content tends to be the preserve of highly educated people, whilst social entertainment and is more common amongst low status social groups. Blank concluded from this data that digital networks were likely to enhance the status and mobility chances of low status individuals who created social and entertainment content. In contrast, digital networks were more likely to constrict the political domain.

However, as Dyson [8] observes, the introduction of technology into a society is likely to transform the society in unanticipated ways as well. For instance, he gives an example of how the advent of the internet transformed rural areas by enabling social elites to move out into the countryside and establish their businesses there. This had the effect of raising property prices in the countryside, thereby bringing about a positive impact in the economic circumstances of rural people. In the same light, $5 \mathrm{G}$, alongside the IoT, has the potential to transform society in hitherto unknown ways. It is quite possible that some socio-economic attributes that give competitive advantages today may lose their relevance, and other socio-economic attributes may emerge as new forms of competitive advantage. Like all new technologies, 5G will bring immense socio-economic changes that have the potential to significantly redraw current social structures.

\section{CONCLUDING REMARKS}

$5 \mathrm{G}$ promises significant economic advantages and considerable social progress. Coupled with the Internet of Things, it is beyond doubt that $5 \mathrm{G}$ will alleviate poverty in those countries that adopt it, as well as reducing social inequality through democratising social opportunities. This is likely to lead to improved quality of life for all. However, 5G also has the potential to substantially reorganise society as we know it. Social transformation involves the unmaking of social elites and the making of new social elites in their place. As such, social transformation is always a painful process, no 
matter how desirable. The challenge for political leaders and policy makers is to anticipate the likelihood of social transformation and to mitigate any trauma associated with it.

5G stands to give an economic advantage to those countries that adopt it first, and whilst economic competition may not be a zero-sum game, it's likely that late adopters are likely to significantly lose out economically and socially. In general technologies tend to be developed for the economic elite. Consequently, it is very likely that the more economically developed countries are more likely to implement 5G first. After all, most of the research towards $5 \mathrm{G}$ is taking place predominantly in the developed countries. Hence, this has the potential to widen the economic gap between well developed and least- developed countries. However, this is not a cast-iron rule, and nimble and deft least developed countries can take the opportunity to leap-frog over their more economically developed counterparts.

\section{REFERENCES}

[1] M. S. Rony and A. J. Pullin, "Design Considerations for 5G Mobile Network," International Journal of Computer Applications, vol. 62, 2013.

[2] A. Gupta and R. K. Jha, "A survey of 5G network: architecture and emerging technologies," IEEE access, vol. 3, pp. 1206-1232, 2015.

[3] M. Agiwal, A. Roy, and N. Saxena, "Next generation 5G wireless networks: A comprehensive survey," IEEE Communications Surveys \& Tutorials, vol. 18, no. 3, pp: 1617-1655, 2016.

[4] J. F. Monserrat, G. Mange, V. Braun, H. Tullberg, G. Zimmermann, and Ö. Bulakci, "METIS research advances towards the $5 \mathrm{G}$ mobile and wireless system definition," EURASIP Journal on Wireless Communications and Networking, vol. 2015, p. 1, 2015.

[5] E. Liotou, H. Elshaer, R. Schatz, R. Irmer, M. Dohler, N. Passas, and L. Merakos, "Shaping QoE in the 5G ecosystem," In Proceedings of Seventh International Workshop on Quality of Multimedia Experience (QoMEX), 2015, pp. 1-6.

[6] Enders Analysis, "TNS RI-Survey," May, 2014.

[7] J. V. Dijk and K. Hacker, "The digital divide as a complex and dynamic phenomenon," The Information Society, vol. 19, pp. 315-326, 2003.

[8] F. Dyson, "Technology and social justice," Carnigie
Concil on Ethics and International Affairs, pp. 7-25, 1997.

[9] E. Hargittai, "The digital reproduction on inequality," in Social Stratification: Class, Race, and Gender in Sociological Perspective, D. Grusky, Ed., ed Boulder, Colorado: Westview Press, 2008, pp. 936-944.

[10] E. Hargittai and Y. P. Hsieh, "Digital Inequality," The Oxford handbook of internet studies, pp. 129-150, 2013.

[11] P. DiMaggio and E. Hargittai, "From the 'digital divide' to 'digital inequality': Studying Internet use as penetration increases," Princeton: Center for Arts and Cultural Policy Studies, Woodrow Wilson School, Princeton University, vol. 4, pp. 4-2, 2001.

[12] B. Wessels, "The reproduction and reconfiguration of inequality: Differentiation and class, status and power in the dynamics of digital divides," The digital divide: The Internet and social inequality in international perspective, pp. 17-28, 2013.

[13] M. Ragnedda and G. W. Muschert, The digital divide: The internet and social inequality in international perspective vol. 73: Routledge, 2013.

[14] J. Van Dijk, "A theory of the digital divide," in The digital divide: the internet and social inequality in international perspective. vol. 73, M. Ragnedda and G. W. Muschert, Eds., ed: Routledge, 2013, pp. 29-51.

[15] S. Oviatt, "Multimodal interfaces," The human-computer interaction handbook: Fundamentals, evolving technologies and emerging applications, vol. 14, pp. 286-304, 2003.

[16] Apple. (2016). Available: http://www.apple.com/uk/ios/siri/

[17] Google. (2016). Google Now. Available: https://www.google.co.uk/landing/now/

[18] L. Atzori, A. Iera, and G. Morabito, "The Internet of Things: A survey," Computer Networks, vol. 54, pp. 2787-2805, 2010.

[19] N. K. Chebib and R. M. Sohail, "The reasons social media contributed to the 2011 Egyptian revolution," International Journal of Business Research and Management (IJBRM), vol. 2, pp. 139-162, 2011.

[20] G. Blank, "Who creates content? Stratification and content creation on the Internet," Information, Соттиnication \& Society, vol. 16, pp. 590-612, 2013.

(C) Author(s) 2016. CC Attribution 4.0 License. (http://creativecommons.org/licenses/by-nc/4.0/)

This article is licensed under the terms of the Creative Commons Attribution Non-Commercial License which permits unrestricted, non-commercial use, distribution and reproduction in any medium, provided the work is properly cited. 